\title{
AN AGE-DEPENDENT POPULATION EQUATION WITH DIFFUSION AND DELAYED BIRTH PROCESS
}

\author{
G. FRAGNELLI
}

Received 2 June 2004 and in revised form 15 June 2005

We propose a new age-dependent population equation which takes into account not only a delay in the birth process, but also other events that may take place during the time between conception and birth. Using semigroup theory, we discuss the well posedness and the asymptotic behavior of the solution.

\section{Introduction}

In this paper, we study an age-dependent population equation where the birth process contains a delay. More precisely, we consider the equation

$$
\begin{gathered}
u_{t}(t, a, x)=-u_{a}(t, a, x)-\mu(a) u(t, a, x)+\Delta_{x} u(t, a, x), \quad t \geq 0, x \in \Omega, a \geq 0, \\
u(s, a, x)=u^{0}(s, a, x), \quad s \in(-\tau, 0], x \in \Omega, a \geq 0, \\
u(0, a, x)=f(a, x), \quad x \in \Omega, a \geq 0, \\
u(t, 0, x)=\int_{0}^{\infty} \int_{-\tau}^{0} \beta(\sigma, a) \tilde{u}(t+\sigma, a, x) d \sigma d a, \quad t \geq 0, x \in \Omega, \\
u(t, a, x)=0 \quad\left(\text { or } \frac{\partial}{\partial \nu} u(t, a, x)=0\right), \quad t>0, a \geq 0, x \in \partial \Omega,
\end{gathered}
$$

where $u(t, a, x)$ represents the density of the population of age $a>0$ at time $t$ and position $x \in \Omega, \Omega$ is a bounded open subset of $\mathbb{R}^{n}, \mu \geq 0$ is the death rate, $\beta \geq 0$ is the birth rate, and $u^{0}$ and $f$ are given functions. The delay operator $\Phi \in \mathscr{L}\left(L^{1}\left((-\tau, 0], L^{1}\left(\mathbb{R}_{+}, X\right)\right), X\right)$, defined as

$$
\Phi F:=\int_{0}^{\infty} \int_{-\tau}^{0} \beta(\sigma, a) F(\sigma, a) d \sigma d a,
$$

is called the birth process. Here $X$ is a general Banach space. Moreover, the modified 
3274 Diffusion and delayed birth in an age population equation

history function $\tilde{u}$ is defined as

$$
\tilde{u}_{t}(s):=\tilde{u}(t+s, a):= \begin{cases}\tilde{V}(s, t+s) u(t+s, a) & \text { for } t+s>0 \\ \widetilde{V}(s, t+s) F(t+s, a) & \text { otherwise }\end{cases}
$$

where $s \in(-\tau, 0], \tau>0$ is the maximal delay, $F$ is a fixed function in the functional space $\mathscr{F}_{\mathcal{F}}:=L^{1}\left((-\tau, 0], L^{1}\left(\mathbb{R}_{+}, X\right)\right)$, and the evolution family $(\tilde{V}(\varsigma, s))_{\sigma \leq s}$ is the trivial extension (see Definition 2.5) of a given evolution family $(V(\varsigma, s))_{-\tau<\varsigma \leq s \leq 0}$, which describes the time lag between conception and birth.

In [22], Piazzera considered a model close to (1.1), but with $u$ independent of $x$, that is, $u(t, a, x):=u(t, a)$. In particular, in [22], the birth process is given by

$$
t \longmapsto \Phi u_{t},
$$

where the history function $u_{t}, t \geq 0$, is defined as

$$
(-\tau, 0] \ni s \longmapsto u_{t}(s, a):= \begin{cases}u(t+s, a), & t+s \geq 0, \\ F(t+s, a), & t+s<0,\end{cases}
$$

and the delay operator $\Phi: L^{1}\left((-\tau, 0] \times \mathbb{R}_{+}\right) \rightarrow \mathbb{R}$ is defined formally as in (1.2), assuming that the birth rate $\beta$ belongs to $L^{\infty}\left(\mathbb{R}_{+}\right)$. In [14], the same model of [22] in analyzed, but in [14], the birth process is given by

$$
u(t, 0)=\int_{0}^{\infty} \beta(a) u(t, a) d a, \quad t \geq 0 .
$$

For further references, see the monographs $[15,26]$.

The real starting point for the present paper is the following model studied in [21]:

$$
\begin{gathered}
u_{t}(t, a, x)=-u_{a}(t, a, x)-\mu(a) u(t, a, x)+\Delta_{x} u(t, a, x), \quad t \geq 0, x \in \Omega, a \geq 0, \\
u(0, a, x)=f(a, x), \quad x \in \Omega, a \geq 0, \\
u(t, 0, x)=\int_{0}^{\infty} \beta(\sigma, a) u(t, a, x) d a, \quad t \geq 0, x \in \Omega, \\
u(t, a, x)=0 \quad \text { or } \quad\left(\frac{\partial}{\partial \nu} u(t, a, x)=0\right), \quad t>0, a \geq 0, x \in \partial \Omega,
\end{gathered}
$$

where the birth process is given by

$$
t \longmapsto \int_{0}^{\infty} \beta(\sigma, a) u(t, a, x) d a, \quad t \geq 0, x \in \Omega .
$$

As we can see, (1.7) does not take into account the fact that a lot of things may happen in the period between conception and birth, for example pregnant individuals can die or can move during the period of gestation and therefore can bear in a place different from that they were fecundated (see, e.g., [13] or [10]). Thus, in the birth process, we have not only to consider the density of the population dependent on the time $t$, on the age $a$, and on the space $x$, but we also have to modify it in some way. To be more precise, we have to 
consider a modified history function. In particular, to include the previous phenomena in the previous model, we have to suppose that the operators which govern the evolution in the past are given by

$$
\Delta_{x}-\mu(\sigma), \quad \sigma \in[0, \tau)
$$

The backward evolution family $(V(s, s))_{-\tau<\varsigma \leq s \leq 0}$ solving the nonautonomous Cauchy problem associated to these operators is

$$
V(\varsigma, s):=e^{-\int_{-s}^{-\varsigma} \mu(\rho) d \rho} e^{(s-\varsigma) \Delta_{x}}
$$

for $\varsigma \leq s \in(-\tau, 0]$ (see below).

This is the reason why we substitute the birth process considered in [21] with

$$
t \longmapsto \int_{0}^{\infty} \int_{-\tau}^{0} \beta(\sigma, a) \tilde{u}(t+\sigma, a, x) d \sigma d a, \quad t \geq 0
$$

where $\tilde{u}(t+\sigma, a)$ is defined as in (1.3). It is important to observe that the term

$$
\int_{0}^{\infty} \int_{-\tau}^{0} \beta(\sigma, a) \tilde{u}(t+\sigma, a, x) d \sigma d a
$$

can be rewritten, using the definition of the backward evolution family $(V(\varsigma, s))_{-\tau<\varsigma \leq s \leq 0}$, as

$$
\int_{0}^{\infty} \int_{-\tau}^{0} e^{\Delta_{x} \sigma} \Pi(-\sigma) \beta(\sigma, a) u(t+\sigma, a, x) d \sigma d a
$$

Here

$$
\Pi(a):=e^{-\int_{0}^{a} \mu(s) d s}, \quad a \geq 0,
$$

denotes the probability of survival up to age $a$. Thanks to the existence of this term, the model (1.1) proposed and studied in this paper is new and more realistic than the models presented, for example, in $[21,22]$.

The paper is organized as follows. In Section 2, we study the evolution in the past. In Section 3, we show how our problem fits into a semigroup framework, and we study the well posedness of the problem using operator matrices theory. In Section 4, we analyze the asymptotic behavior of the solution of problem (1.1). In particular, we give a condition such that the solution of (1.1) decays exponentially. This is important if $u$ represents a virus. However, until now, we cannot say anything about the asymptotic behavior of the solution if the previous condition is not satisfied. For this reason, it is interesting to control (1.1) in some way (see, e.g., $[1,2]$ or $[4])$. For this problem, we refer to a forthcoming paper. 


\section{Derivation of the equation and evolution in the past}

Consider the following linear age-dependent population equation with delayed birth process:

$$
\begin{gathered}
u_{t}(t, a, x)=-u_{a}(t, a, x)-\mu(a) u(t, a, x)+\Delta_{x} u(t, a, x), \quad x \in \Omega, a \geq 0, \\
u(s, a, x)=u^{0}(s, a, x), \quad s \in(-\tau, 0], x \in \Omega, a \geq 0, \\
u(t, 0, x)=\int_{0}^{\infty} \int_{-\tau}^{0} \beta(\sigma, a) v(t, a, \sigma, x) d \sigma d a, \quad t \geq 0, \\
u(t, a, x)=0 \quad \text { or } \quad\left(\frac{\partial}{\partial \nu} u(t, a, x)=0\right), \quad t>0, a \geq 0, x \in \partial \Omega,
\end{gathered}
$$

where $\Omega$ is a bounden, open subset of $\mathbb{R}^{n}$, and $v(t, a, \sigma, x)$ is the density of the subpopulation collecting pregnant individuals of age $a$, with time of gestation $\sigma$, that at time $t$ are at the position $x$. Therefore, assume that the density of the subpopulation $v(t, a, \sigma, x)$ is governed by the following operators:

$$
B(\sigma):=\Delta_{x}-\mu(\sigma)
$$

that is,

$$
v_{t}(t, a, \sigma, x)=-v_{\sigma}(t, a, \sigma, x)-\mu(\sigma) v(t, a, \sigma, x)+\Delta_{x} v(t, a, \sigma, x) .
$$

On the nonnegative death and birth rates, we make the following assumptions:

$$
\begin{gathered}
\mu \in L_{\mathrm{loc}}^{\infty}\left(\mathbb{R}_{+}\right), \quad \beta \in L^{\infty}\left((-\tau, 0] \times \mathbb{R}_{+}\right), \\
\inf _{a \in[0, \infty)} \mu(a)=: \mu_{\infty}>0 .
\end{gathered}
$$

Here $\Delta_{x}$ is the Laplace operator on $\Omega$ and $\partial / \partial \nu$ is the outward normal derivative. Thus, we assume that $\Omega$ is arbitrary in the case of Dirichlet boundary conditions and that $\Omega$ has the extension property otherwise. Here $D\left(\Delta_{x}\right)$ denotes the domain of the Laplacian on $X:=L^{1}(\Omega)$. Moreover, set $E:=L^{1}\left(\mathbb{R}_{+}, X\right)$, which is the natural state space for $(1.1)$ because the $L^{1}$-norm of $u$ gives the total population size. (We recall here that the Laplace operator $\left(\Delta_{x}, D\left(\Delta_{x}\right)\right)$ with Dirichlet (or Neumann) boundary conditions on an arbitrary open subset $\Omega$ of $\mathbb{R}^{n}$ (which has the extension property in presence of Neumann boundary conditions, see, e.g., [5]) generates an analytic strongly continuous semigroup (see, e.g., [3]).)

As we saw in the introduction, the backward evolution family $(V(\varsigma, s))_{-\tau<\varsigma \leq s \leq 0}$ solving the nonautonomous Cauchy problem associated to the operators $B(\sigma)$ is given by

$$
V(\varsigma, s):=e^{-\int_{-s}^{-\varsigma} \mu(\rho) d \rho} e^{(s-\varsigma) \Delta_{x}}
$$

for all $\varsigma \leq s \in(-\tau, 0]$ (see, e.g., [20] or [25]). This takes into account the fact that, in general, pregnant individuals can move during the period of gestation, bearing in a place different from that they were fecundated, and that, therefore, they can die. 
Proceeding as in [13], one can prove that

$$
\int_{0}^{\infty} \int_{-\tau}^{0} \beta(\sigma, a) v(t, a, \sigma, x) d \sigma d a=\int_{0}^{\infty} \int_{-\tau}^{0} \beta(\sigma, a) \tilde{u}(t+\sigma, a, x) d \sigma d a .
$$

Thus system (2.1) can be rewritten as (1.1).

Before continuing, we will recall some definitions (see, e.g., [19]) and results.

Definition 2.1. A family $(V(\varsigma, s))_{\varsigma \leq s \leq 0}$ of bounded, linear operators on a Banach space $X$ is called an (exponentially bounded, backward ) evolution family if

(i) $V(\varsigma, r) V(r, s)=V(\varsigma, s), V(\varsigma, \varsigma)=$ Id for all $\varsigma \leq r \leq s \leq 0$,

(ii) the mapping $(\varsigma, s) \mapsto V(\varsigma, s)$ is strongly continuous,

(iii) $\|V(\varsigma, s)\| \leq M_{\omega} e^{\omega(s-\varsigma)}$ for some $M_{\omega} \geq 1, \omega \in \mathbb{R}$ and all $\varsigma \leq s \leq 0$.

Definition 2.2. Let $\mathscr{V}:=(V(\varsigma, s))_{\varsigma \leq s \leq 0}$ be a backward evolution semigroup. Define the growth bound of $\mathscr{V}$ as

$$
\omega_{0}(\mathscr{V}):=\inf \left\{\omega \in \mathbb{R}: \exists M_{\omega} \geq 1 \text { with }\|V(\varsigma, s)\| \leq M_{\omega} e^{\omega(s-\varsigma)}, \forall \varsigma \leq s \leq 0\right\} .
$$

In particular, for the evolution family defined in (2.5), the next property holds.

Proposition 2.3. The growth bound of the backward evolution family defined in (2.5) is negative. In particular,

$$
\omega_{0}(\mathscr{V})=\omega_{0}(T(\cdot))-\mu_{\tau}<0
$$

where $(T(t))_{t \geq 0}$ is the semigroup generated by the Laplace operator $\Delta_{x}$ and $\mu_{\tau}:=$ $\inf _{\rho \in[0, \tau)} \mu(\rho)$.

Now, consider the backward nonautonomous Cauchy problem

$$
\begin{gathered}
\dot{u}(\varsigma)=-B(\varsigma) u(\varsigma), \quad-\tau<\varsigma \leq s \leq 0, \\
u(s)=f \in E,
\end{gathered}
$$

on a general Banach space 9 for a family $(B(\varsigma), D(B(\varsigma)))_{\varsigma \in(-\tau, 0]}$ of (unbounded) linear operators.

Definition 2.4. The problem (NCP) is said to be well posed with regularity subspaces $\left(Y_{s}\right)_{s \in(-\tau, 0]}$ if the following conditions hold.

(i) Existence. For all $s \in(-\tau, 0]$, the subspace

$$
Y_{s}:=\{f \in E: \text { there exists a classical solution for }(\mathrm{NCP})\} \subset D(B(s))
$$

is dense in $E$.

(ii) Uniqueness. For every $f \in Y_{s}$, the solution $u_{s}(\cdot, f)$ of (NCP) is unique.

(iii) Continuous dependence. The solutions depend continuously on $s$ and $f$, that is, if $s_{n} \rightarrow s \in(-\tau, 0], f_{n} \rightarrow f \in Y_{s}$ with $f_{n} \in Y_{s_{n}}$, then

$$
\left\|\widehat{u}_{s_{n}}\left(\varsigma, f_{n}\right)-\widehat{u}_{s}(\varsigma, f)\right\| \longrightarrow 0
$$


uniformly for $\sigma$ in compact subsets of $(-\tau, 0]$, where

$$
\hat{u}_{s}(\varsigma, f):= \begin{cases}u_{s}(\varsigma, f) & \text { if } s \geq \varsigma \\ f & \text { if } s<\varsigma .\end{cases}
$$

If, in addition, there exist constants $\omega \in \mathbb{R}$ and $M_{\omega} \geq 1$ such that

$$
\left\|u_{s}(\varsigma, f)\right\| \leq M_{\omega} e^{\omega(s-\varsigma)}\|f\|
$$

for all $f \in Y_{s}$ and $t \geq s$, then (NCP) is called well posed with exponentially bounded solutions.

As in [20, Proposition 2.5], we can show that for each well-posed (NCP), there exists a unique backward evolution family $(V(s, s))_{-\tau<\varsigma \leq s \leq 0}$ solving (NCP), that is, the function $\varsigma \mapsto u(\varsigma):=V(\varsigma, s) f$ is a classical solution of (NCP) for $s \in(-\tau, 0]$ and $f \in Y_{s}$.

In this paper, we will use evolution semigroup techniques for which we refer to [8, Section VI.9]. To this purpose, we first extend $(V(\varsigma, s))_{-\tau<\varsigma \leq s \leq 0}$ to an evolution family $(\tilde{V}(\varsigma, s))_{\varsigma \leq s}$ on $\mathbb{R}$ (see, e.g., [11]).

Definition 2.5. (1) The evolution family $(V(\varsigma, s))_{-\tau<\varsigma \leq s \leq 0}$ on $E$ is extended to an evolution family $(\tilde{V}(\varsigma, s))_{\varsigma \leq s}$ by setting

$$
\tilde{V}(\varsigma, s):= \begin{cases}V(\varsigma, s) & \text { for }-\tau<\varsigma \leq s \leq 0 \\ V(\varsigma, 0) & \text { for }-\tau<\varsigma \leq 0 \leq s \\ V(0, s) & \text { for } \varsigma<-\tau<s \leq 0 \\ V(0,0)=\text { Id } & \text { otherwise. }\end{cases}
$$
by

(2) On the space $\tilde{\mathscr{F}}:=L^{1}(\mathbb{R}, E)$, define the corresponding evolution semigroup $(\tilde{S}(t))_{t \geq 0}$

$$
(\widetilde{S}(t) \widetilde{F})(s):=\tilde{V}(s, s+t) \widetilde{F}(s+t)= \begin{cases}V(s, s+t) \tilde{F}(s+t) & \text { for }-\tau<s \leq s+t \leq 0, \\ V(s, 0) \tilde{F}(s+t) & \text { for }-\tau<s \leq 0 \leq s+t \\ V(0, s+t) \widetilde{F}(s+t) & \text { for } s<-\tau<s+t \leq 0, \\ \widetilde{F}(s+t) & \text { otherwise, }\end{cases}
$$

for all $\widetilde{F} \in \tilde{\mathscr{F}}, s \in \mathbb{R}, t \geq 0$.

It is easy to prove that the semigroup $(\tilde{S}(t))_{t \geq 0}$ is strongly continuous on $\tilde{\mathscr{F}}_{\text {fee }}[8$, Lemma VI.9.10]). We denote its generator by $(\widetilde{G}, D(\widetilde{G}))$. Note that the precise description of the domain $D(\widetilde{G})$ is difficult.

Moreover, since $(\widetilde{G}, D(\widetilde{G}))$ is a local operator (see [23, Theorem 2.4]), we can restrict it to the space $\mathscr{F}:=L^{1}((-\tau, 0], E)$ by the following definition. 
Definition 2.6. Take

$$
D(G):=\left\{\widetilde{F}_{\left.\right|_{(-\tau, 0]}}: \tilde{F} \in D(\widetilde{G})\right\}
$$

and define

$$
G F:=(\tilde{G} \widetilde{F})_{\mid(-\tau, 0]} \quad \text { for } F=\widetilde{F}_{\left.\right|_{(-\tau, 0]}} \in D(\widetilde{G})
$$

The operator $G$ is not a generator on $\mathscr{F}$. However, if we identify $\mathscr{F}$ with the subspace $\left\{F \in \widetilde{\mathscr{F}}_{\mathcal{F}}: F(s)=0 \forall s \in(-\infty,-\tau] \cup[0,+\infty)\right\}$, then $\mathscr{F}_{\mathcal{F}}$ remains invariant under $(\tilde{S}(t))_{t \geq 0}$. As a consequence, we obtain the following lemma.

Lemma 2.7. The semigroup $\left(S_{0}(t)\right)_{t \geq 0}$ induced by $(\tilde{S}(t))_{t \geq 0}$ on $\mathscr{F}$ is

$$
\left(S_{0}(t) F\right)(s)= \begin{cases}V(s, s+t) F(t+s) & \text { for }-\tau<s+t \leq 0 \\ 0 & \text { otherwise }\end{cases}
$$

for any $F \in \mathscr{F}$.

The following lemma characterizes the generator of this semigroup. Lemma 2.8 (see [12]). The generator $\left(G_{0}, D\left(G_{0}\right)\right)$ of $\left(S_{0}(t)\right)_{t \geq 0}$ is given by

$$
D\left(G_{0}\right)=\{F \in D(\widetilde{G}) \cap \mathscr{F}: F(0)=0\}, \quad G_{0} F=G F
$$

We thus end up with operators $\left(G_{0}, D\left(G_{0}\right)\right) \subset(G, D(G)) \subset(\widetilde{G}, D(\widetilde{G}))$, where only the first and the third are generators.

Remark 2.9. Observe that $G_{0}=G_{\left.\right|_{\mathrm{KerL}}}$, where $L: D(G) \rightarrow E$ is such that $L F=F(0)$.

Moreover, as in [9], one can prove that, for all $\lambda \in \mathbb{C}$,

$$
\operatorname{Ker}(\lambda-G)= \begin{cases}\left\langle\epsilon_{\lambda}\right\rangle & \text { for } \mathfrak{R} \lambda>\omega_{0}(\mathscr{V}) \\ \{0\} & \text { otherwise }\end{cases}
$$

where the bounded linear operators $\epsilon_{\lambda}: E \rightarrow \mathscr{F}$ are defined as

$$
\left(\epsilon_{\lambda} f\right)(s):=e^{\lambda s} V(s, 0) f, \quad-\tau \leq s \leq 0, f \in E .
$$

Therefore, following, for example, [16, Theorem 2.3], one can prove that the spectral mapping theorem holds for $\left(S_{0}(t)\right)_{t \geq 0}$. 
Theorem 2.10. Let $\left(G_{0}, D\left(G_{0}\right)\right)$ be the generator of $\left(S_{0}(t)\right)_{t \geq 0}$ on $F_{\text {f }}$. Then the spectrum of $\left(S_{0}(t)\right)_{t \geq 0}, \sigma\left(S_{0}(t)\right)$, is a disk centered at the origin and the spectrum $\sigma\left(G_{0}\right)$ of $G_{0}$ is a half-plane. Moreover, $\left(S_{0}(t)\right)_{t \geq 0}$ satisfies the spectral mapping theorem

$$
\sigma\left(S_{0}(t)\right) \backslash\{0\}=e^{t \sigma\left(G_{0}\right)}, \quad t \geq 0 .
$$

In particular, $s\left(G_{0}\right)=\omega_{0}\left(S_{0}(\cdot)\right)=\omega_{0}(\mathscr{V})$. Here $s\left(G_{0}\right)$ is the spectral bound of $G_{0}$, defined as

$$
s\left(G_{0}\right):=\sup \left\{\Re \lambda: \lambda \in \sigma\left(G_{0}\right)\right\} .
$$

Thus

$$
\text { if } \lambda \in \mathbb{C} \text { is such that } \mathfrak{R} \lambda>\omega_{0}(\mathscr{V}) \text {, then } \lambda \in \rho\left(G_{0}\right) \text {, }
$$

where $\rho\left(G_{0}\right)$ is the resolvent set of $G_{0}$, that is,

$$
\rho\left(G_{0}\right):=\left\{\lambda \in \mathbb{C}: \text { s.t. }\left(\lambda-G_{0}\right) \text { is invertible }\right\} .
$$

\section{Well posedness}

This section is devoted to studying the well posedness of (1.1), that is, to proving the existence of a solution of (1.1). To do this, the main idea is to use semigroup theory. In particular, we will rewrite the model as an abstract Cauchy problem of the type

$$
\begin{aligned}
\dot{u}(t) & =\mathscr{A} u(t), \quad t \geq 0, \\
u(0) & =u_{0},
\end{aligned}
$$

and then we will apply the following result due to G. Greiner.

Let $X, \partial X$ be two Banach spaces and $\mathscr{A}:(D(\mathscr{A}),|\cdot|) \rightarrow X$ and $L:(D(\mathscr{A}),|\cdot|) \rightarrow \partial X$ two continuous linear operators such that $(D(\mathscr{A}),|\cdot|)$ is complete and $\operatorname{Im} L=\partial X$. Moreover, let $\mathscr{A}_{\Phi}$ be the operator defined as follows:

$$
\mathscr{A}_{\Phi} x:=\mathscr{A} x, \quad D\left(\mathscr{A}_{\Phi}\right):=\{x \in D(\mathscr{A}): L x=\Phi x\}
$$

for a bounded operator $\Phi: X \rightarrow \partial X$. Then the next theorem holds.

Theorem 3.1 (see [14]). If $\mathscr{A}_{0}:=\mathscr{A}_{\left.\right|_{\mathrm{KerL}}}$ generates a strongly continuous semigroup $\left(T_{0}(t)\right)_{t \geq 0}$ on $X$ and there exist constants $\gamma>0$ and $\lambda_{0} \in \mathbb{R}$ such that for every $\lambda>\lambda_{0}$ the following condition is satisfied:

$$
\left\|L_{\lambda}\right\| \leq(\lambda \gamma)^{-1}
$$

then $\mathscr{A}_{\Phi}$ is a generator. Here $L_{\lambda}:=\left(L_{\left.\right|_{\operatorname{Ker}(\lambda-s l)}}\right)^{-1}$, where $\lambda \in \rho\left(\mathscr{A}_{0}\right)$.

Hence, the first step is to rewrite (1.1) as an abstract Cauchy problem. To this aim, we will prove, first of all, the equivalence of (1.1) with an appropriate boundary delay problem. 
3.1. First step: (1.1) as an abstract boundary delay problem. In this subsection, we want to rewrite (1.1) as an abstract boundary delay problem. At first we consider the following subspace $\mathscr{D}_{0}$ of $E$ defined as $\mathscr{D}_{0}:=D_{0} \cap D_{\Delta_{x}}$, where

$$
\begin{gathered}
D_{0}:=\left\{f \in E: f(\cdot, x) \text { is continuous and a.e. differentiable on } \mathbb{R}_{+},\right. \\
\left.f(0, x)=0 \text { for almost all } x \in \Omega, \frac{\partial f}{\partial a}+\mu f \in E\right\}, \\
D_{\Delta_{x}}:=\left\{f \in E: f(a, \cdot) \in D\left(\Delta_{x}\right), \forall a \geq 0, \Delta_{x} f \in E\right\} .
\end{gathered}
$$

As in [21], we consider the family of linear operator $A$ on $X$ given by

$$
(A f)(a):=-\mu(a) f(a)+\Delta_{x} f(a)
$$

and, as in [22], the map $P: D(A) \rightarrow X$, defined as $P f:=f(0)$ and called the boundary operator. The following proposition holds.

Proposition 3.2. If $u \in \mathscr{D}_{0},(1.1)$ is equivalent to the following abstract boundary delay problem:

$$
\begin{aligned}
& \dot{u}(t)=A u(t), \\
& P u(t)=\Phi \tilde{u}_{t}, \\
& \tilde{u}_{0}=u^{0}, \\
& u(0)=f,
\end{aligned}
$$

where $f \in E$ and $u^{0} \in \mathscr{F}_{\text {. }}$. Moreover, the function $u:[0,+\infty) \rightarrow E$ is defined as $u(t):=$ $u(t, \cdot, x)$ and $\tilde{u}_{t}:(-\tau, 0] \rightarrow E$ and $\Phi$ are defined as in (1.3) and (1.2), respectively.

Proof. Let $\left(A_{0}, D\left(A_{0}\right)\right)$ be the operator defined as $A_{0} f:=A f$ with $D\left(A_{0}\right):=\{f \in D(\tilde{A}) \cap$ $E: f(0)=0\}((\tilde{A}, D(\tilde{A}))$ is the natural extension of $(A, D(A))$, see, e.g., Section 2). It is easy to prove that $A_{0}:=A_{\mid \mathrm{KerP}}$ and, as in [14], that it generates the following evolution semigroup on $E$ :

$$
T_{0}(t) f(a)= \begin{cases}U(a, a-t) f(a-t), & a \geq t, \\ 0, & a<t,\end{cases}
$$

where $(U(t, s))_{t \geq s \geq 0}$ is the forward evolution family

$$
U(t, s)=e^{-\int_{s}^{t} \mu(\sigma) d \sigma} e^{(t-s) \Delta_{x}}
$$

for $t \geq s \geq 0$. As in [21, Lemma 5.1], one can prove that $\mathscr{D}_{0}$ is a core of $A_{0}$ and

$$
\left(A_{0} f\right)(a)=-\frac{\partial}{\partial a} f(a)-\mu(a) f(a)+\Delta_{x} f(a),
$$

for every $f \in \mathscr{D}_{0}$ and a.e. $a \geq 0$ (recall that a core $D$ of a generator $(A, D(A)$ ) is a subspace of $D(A)$ which is dense in the graph norm $\|x\|_{A}:=\|x\|+\|A x\|, x \in D(A)$ ).

Thus the thesis follows immediately, observing that $P u(t)=u(t, 0)=\Phi \tilde{u}_{t}$. 
3282 Diffusion and delayed birth in an age population equation

3.2. Second step: (3.6) as an abstract Cauchy problem. Here we rewrite (3.6) as an abstract Cauchy problem. To this aim, we define on the product space $\mathscr{E}:=\mathscr{F} \times E$ the operator matrix

$$
\mathscr{A}_{m}:=\left(\begin{array}{cc}
G & 0 \\
0 & A
\end{array}\right),
$$

with maximal domain $D\left(\mathscr{A}_{m}\right):=D(G) \times D(A)$ and define also the operator $\mathscr{L}: D\left(\mathscr{A}_{m}\right) \rightarrow$ $\partial \mathscr{E}:=E \times X$ as the matrix

$$
\mathscr{L}:=\left(\begin{array}{ll}
L & 0 \\
0 & P
\end{array}\right) .
$$

Since $L$ and $P$ are surjective, then the following proposition is immediate.

Proposition 3.3. The operator $\mathscr{L}$ is surjective.

Finally, define the delay operator matrix $\Psi: \mathscr{E} \rightarrow \partial \mathscr{E}$ as

$$
\Psi:=\left(\begin{array}{cc}
0 & \operatorname{Id}_{E} \\
\Phi & 0
\end{array}\right)
$$

and the operator $\mathscr{A}$ as

$$
\mathscr{A}:=\mathscr{A}_{m_{\mid \mathrm{Ker}(\mathscr{L}-\Psi)}} .
$$

The following definition is quite natural.

Definition 3.4. A continuous function $u:(-\tau,+\infty) \rightarrow X$ is called a classical solution of (3.6) with initial value $\left(\begin{array}{c}u^{0} \\ f\end{array}\right) \in D(\mathscr{A})$ if it is continuously differentiable on $[0,+\infty), u(t) \in$ $D(A), \tilde{u}_{t} \in D(G)$ for all $t \geq 0$, and if it satisfies (3.6).

The next proposition holds.

Proposition 3.5. If the function $U: t \in[0,+\infty) \mapsto \vartheta(t)$ defined as

$$
u(t):=\left(\begin{array}{c}
\tilde{u}_{t} \\
u(t)
\end{array}\right)
$$

is a solution of

$$
\begin{aligned}
& \dot{u}(t)=\mathscr{A} u(t), \quad t \geq 0, \\
& \dot{u}(0)=\left(\begin{array}{c}
\tilde{u}_{0} \\
u(0)
\end{array}\right),
\end{aligned}
$$

then $u(t):=\Pi_{2}(\mathcal{U}(t))$ solves (3.6).

Proof of Proposition 3.5. The proof is an easy consequence of the fact that the operator $\mathscr{A}$ can be rewritten as

$$
\mathcal{A}:=\left(\begin{array}{ll}
G & 0 \\
0 & A
\end{array}\right),
$$


with domain

$$
\begin{aligned}
D(\mathscr{A}) & :=\left\{\left(\begin{array}{c}
u^{0} \\
f
\end{array}\right) \in D\left(\mathscr{A}_{m}\right): \mathscr{L}\left(\begin{array}{c}
u^{0} \\
f
\end{array}\right)=\Psi\left(\begin{array}{c}
u^{0} \\
f
\end{array}\right)\right\} \\
& =\left\{\left(\begin{array}{c}
u^{0} \\
f
\end{array}\right) \in D(G) \times D(A): L u^{0}=f, P f=\Phi u^{0}\right\} \\
& =\left\{\left(\begin{array}{c}
u^{0} \\
f
\end{array}\right) \in D(G) \times D(A): u^{0}(0)=f, f(0)=\Phi u^{0}\right\} .
\end{aligned}
$$

Using Propositions 3.2 and 3.5, the following proposition is immediate.

Proposition 3.6. If $(\mathscr{A}, D(\mathscr{A}))$ generates a strongly continuous semigroup $(\mathcal{T}(t))_{t \geq 0}$, then $u(t):=\Pi_{2}(U(t))=\Pi_{2}(\mathscr{T}(t) \cup(0))$ solves (3.6). As a consequence, if $u \in \mathscr{D}_{0}$, then $u$ is the unique solution of (1.1).

3.3. Well posedness. In this subsection, we want to prove that the operator $(\mathscr{A}, D(\mathscr{A}))$ is a generator of a strongly continuous semigroup in order to apply Proposition 3.6 and to conclude that model (1.1) has a solution. The main idea is to apply Theorem 3.1. The next result holds.

Theorem 3.7. The operator $(\mathscr{A}, D(\mathscr{A})$ ) generates a strongly continuous semigroup $(\mathscr{T}(t))_{t \geq 0}$.

\section{Proof}

Step 1. First of all, define the operator $\mathscr{A}_{0}:=\mathscr{A}_{m_{\mid \mathrm{Ker}} \mathscr{S}}$, that is,

$$
\begin{gathered}
\mathscr{A}_{0}:=\left(\begin{array}{cc}
G_{0} & 0 \\
0 & A_{0}
\end{array}\right), \\
D\left(\mathscr{A}_{0}\right)=\left\{\left(\begin{array}{c}
u^{0} \\
f
\end{array}\right) \in D\left(\mathscr{A}_{m}\right): \mathscr{L}\left(\begin{array}{c}
u^{0} \\
f
\end{array}\right)=0\right\} \\
=\left\{\left(\begin{array}{c}
u^{0} \\
f
\end{array}\right) \in D(G) \times D(A): u^{0}(0)=0, f(0)=0\right\} \\
=D\left(G_{0}\right) \times D\left(A_{0}\right) .
\end{gathered}
$$

It is easy to prove that $\left(\mathscr{A}_{0}, D\left(\mathscr{A}_{0}\right)\right)$ generates a strongly continuous semigroup $\left(\mathscr{T}_{0}(t)\right)_{t \geq 0}$ on $\mathscr{E}$ given by

$$
\mathscr{T}_{0}(t)=\left(\begin{array}{cc}
S_{0}(t) & 0 \\
0 & T_{0}(t)
\end{array}\right)
$$

(see [17, Proposition 3.1]).

Step 2. As in Proposition 2.3, one can prove that the growth bound of the evolution family $U:=(U(t, s))_{t \geq s \geq 0}$ is negative. In particular, it is given by

$$
\omega_{0}(U)=\omega_{0}(T(\cdot))-\mu_{\infty}<0,
$$


3284 Diffusion and delayed birth in an age population equation

where $(T(t))_{t \geq 0}$ is the heat semigroup. Note that if we consider the same conditions for the Laplacian in the present and in the past, that is, the Laplacian with Dirichlet or Neumann conditions, then

$$
\omega_{0}(\mathscr{V}) \leq \omega_{0}(\mathscr{U})
$$

since $\mu_{\tau} \geq \mu_{\infty}$. Moreover, as in Section 2, we have that

$$
\text { if } \lambda \in \mathbb{C} \text { is such that } \mathfrak{R} \lambda>\omega_{0}(\mathcal{U}) \text {, then } \lambda \in \rho\left(A_{0}\right) \text {, }
$$

where $\rho\left(A_{0}\right)$ is the resolvent set of $A_{0}$, and

$$
\omega_{0}(\mathcal{U})=\omega_{0}\left(T_{0}(\cdot)\right)=s\left(A_{0}\right) .
$$

An immediate consequence is the following:

$$
\left\{\lambda \in \mathbb{C}: \text { s.t. } \mathfrak{R} \lambda>\max \left\{\omega_{0}(\mathscr{V}), \omega_{0}(\mathscr{U})\right\}\right\} \subseteq \rho\left(G_{0}\right) \cap \rho\left(A_{0}\right) \text {. }
$$

In particular, if $\omega_{0}(\mathscr{V}) \leq \omega_{0}(\mathscr{U})$, then (3.24) becomes

$$
\text { if } \lambda \in \mathbb{C} \text { is such that } \mathfrak{R} \lambda>\omega_{0}(\mathcal{U}) \text {, then } \lambda \in \rho\left(A_{0}\right) \cap \rho\left(G_{0}\right) \text {. }
$$

Therefore, one can prove that

$$
\operatorname{Ker}\left(\lambda-A_{0}\right)= \begin{cases}\left\langle\psi_{\lambda}\right\rangle, & \mathfrak{R} \lambda>\omega_{0}(\mathscr{U}) \\ \{0\} & \text { otherwise }\end{cases}
$$

where the bounded linear operators $\psi_{\lambda}: X \rightarrow E$ are defined as

$$
\left(\psi_{\lambda} y\right)(a):=e^{-\int_{0}^{a}(\lambda+\mu(s)) d s} e^{a \Delta_{x}} y=e^{-\lambda a} U(a, 0) y .
$$

Step 3. Since $\Psi$ is bounded and $\mathscr{L}$ is surjective, $\mathscr{L}_{\left.\right|_{\operatorname{Ker}\left(\lambda-s_{m}\right)}}$ is an isomorphism of $\operatorname{Ker}(\lambda-$ $\left.\mathscr{A}_{m}\right)$ onto $\partial \mathscr{E}$ for $\lambda \in \mathbb{C}$ with $\mathfrak{R} \lambda>\max \left\{\omega_{0}(\mathscr{V}), \omega_{0}(\mathscr{U})\right\}$ (see, e.g., [14]). Thus, we can define $\mathscr{L}_{\lambda}: \partial \mathscr{E} \rightarrow \operatorname{Ker}(\lambda-\mathscr{A})$ as

$$
\mathscr{L}_{\lambda}:=\left(\mathscr{L}_{\left.\right|_{\operatorname{Ker}\left(\lambda-s l_{m}\right)}}\right)^{-1}=\left(\begin{array}{cc}
L_{\lambda} & 0 \\
0 & P_{\lambda}
\end{array}\right),
$$

where $L_{\lambda}: E \rightarrow \operatorname{Ker}(\lambda-G)$ is given by $L_{\lambda}:=\left(L_{\mid \operatorname{Ker}(\lambda-G)}\right)^{-1}$ and $P_{\lambda}: X \rightarrow \operatorname{Ker}(\lambda-A)$ is defined by $P_{\lambda}:=\left(P_{\mid \operatorname{Ker}(\lambda-A)}\right)^{-1}$. Now we want to compute $\mathscr{L}_{\lambda}$. To this aim, it is sufficient to find $L_{\lambda}$ and $P_{\lambda}$.

Let $f \in \operatorname{Ker}(\lambda-A)$. Then, by (3.26), there exists $y \in X$ such that

$$
f=\psi_{\lambda} \otimes y
$$

where

$$
\left(\psi_{\lambda} \otimes y\right)(\sigma)=e^{-\lambda \sigma} U(\sigma, 0) y, \quad \sigma \geq 0
$$


Thus, $P f=f(0)=\left(\psi_{\lambda} \otimes y\right)(0)=y$ and

$$
P_{\lambda}=\psi_{\lambda} \otimes \operatorname{Id}_{X}
$$

Analogously, let $F \in \operatorname{Ker}(\lambda-G)$. By (2.19), there exists $f \in E$ such that

$$
F=\epsilon_{\lambda} \otimes f
$$

where

$$
\left(\epsilon_{\lambda} \otimes f\right)(s)=e^{\lambda s} V(s, 0) f
$$

for $s \in(-\tau, 0]$. Thus, $L F=F(0)=\left(\epsilon_{\lambda} \otimes f\right)(0)=f$ and

$$
L_{\lambda}=\epsilon_{\lambda} \otimes \operatorname{Id}_{E}
$$

Then, if $\lambda \in \mathbb{C}$ is such that $\mathfrak{R} \lambda>\max \left\{\omega_{0}(\mathscr{U}), \omega_{0}(\mathscr{V})\right\}$, it follows that

$$
\mathscr{L}_{\lambda}=\left(\begin{array}{cc}
\epsilon_{\lambda} \otimes \mathrm{Id}_{E} & 0 \\
0 & \psi_{\lambda} \otimes \operatorname{Id}_{X}
\end{array}\right) .
$$

Step 4. In order to apply Theorem 3.1, we have to find two constants $\gamma>0$ and $\lambda_{0} \in \mathbb{R}$ such that (3.3) holds. Thus we have to estimate the norm of $\mathscr{L}_{\lambda}$. To do this, it is sufficient to estimate the norms of $L_{\lambda}$ and $P_{\lambda}$.

Let $\lambda \in \mathbb{R}$ be such that $\lambda>0$. Since $\omega_{0}(\mathcal{U})$ and $\omega_{0}(\mathscr{V})$ are strictly negative, then, by definition of growth bound, taking $\omega:=0$, there exist $M_{0,1} \geq 1$ and $M_{0,2} \geq 1$ such that $\|V(\sigma, s)\| \leq M_{0,1}$ and $\|U(t, \tau)\| \leq M_{0,2}$ for all $\sigma \leq s \leq 0 \leq \tau \leq t$. Now, it is very easy to prove that

$$
\begin{aligned}
\left\|L_{\lambda}\right\|_{\mathscr{F}} & \leq \frac{M_{0}}{\lambda} \\
\left\|P_{\lambda}\right\|_{E} & \leq \frac{M_{0}}{\lambda}
\end{aligned}
$$

where $M_{0}:=\max \left\{M_{0,1}, M_{0,2}\right\} \geq 1$. Hence, if $\lambda \in \mathbb{R}_{+}$, then the norm of $\mathscr{L}_{\lambda}$ satisfies the following estimate:

$$
\left\|\mathscr{L}_{\lambda}\right\|_{\mathscr{E}} \leq \frac{2 M_{0}}{\lambda}
$$

Step 5. Set $\gamma:=1 / 2 M_{0}$, where $M_{0}$ is as before. Then $\gamma$ is strictly positive. Moreover, by the previous step, one has

$$
\left\|\mathscr{L}_{\lambda}\right\|_{\mathscr{E}} \leq \frac{2 M_{0}}{\lambda}=\frac{1}{\lambda \gamma},
$$

for $\lambda>0$. Thus, by Theorem 3.1 applied with $\lambda_{0}:=0$, we have that $(\mathscr{A}, D(\mathscr{A}))$ generates a strongly continuous semigroup $(\mathscr{T}(t))_{t \geq 0}$. 
The next corollary is an immediate consequence of Proposition 3.6 and Theorem 3.7.

Corollary 3.8. System (3.6) has a unique classical solution $u(t)$. As a consequence, if $u(t) \in \mathscr{D}_{0}$, then $u(t)$ is the unique solution of (1.1).

\section{Asymptotic behavior}

In this section we want to study the asymptotic behavior of the solution $u$ of (1.1). Since it is given through the semigroup $(\mathscr{T}(t))_{t \geq 0}$ generated by $(\mathscr{A}, D(\mathscr{A}))$ (see Proposition 3.6), it is clear that the asymptotic behavior of $u$ is related to the asymptotic behavior of $(\mathscr{T}(t))_{t \geq 0}$. Thus we have to find conditions such that the semigroup $(\mathscr{T}(t))_{t \geq 0}$ decays exponentially, that is, the growth bound of $(\mathscr{T}(t))_{t \geq 0}$ is strictly negative. This is important if, for example, $u$ represents a virus.

The main idea here is to use spectral theory in combination with positivity. Indeed, if the semigroup is positive on the space $\mathscr{E}:=L^{1}\left((-\tau, 0], L^{1}\left(\mathbb{R}_{+}, L^{1}(\Omega)\right)\right) \times L^{1}\left(\mathbb{R}_{+}, L^{1}(\Omega)\right)$, which is an AL-space (see [24, Section II.8]), then the spectral bound $s(\mathscr{A})$ coincides with the growth bound $\omega_{0}(\mathscr{T}(\cdot))$ of the semigroup $(\mathscr{T}(t))_{t \geq 0}$.

Thus the next result is very important.

Proposition 4.1. The semigroup $(\mathcal{T}(t))_{t \geq 0}$ generated by the operator $(\mathscr{A}, D(\mathscr{A}))$ (see Theorem 3.7) is positive.

Proof. If we prove that for all $\lambda \in \mathbb{C}$ such that $\mathfrak{R} \lambda$ is big enough the resolvent of $\mathscr{A}$ in $\lambda$ is positive, then as a consequence of the characterization theorem (see $[8$, Theorem VI.1.8]), we have the positivity of the semigroup generated by $(\mathscr{A}, D(\mathscr{A}))$.

To this aim, let $\lambda \in \mathbb{C}$ be such that $\mathfrak{R} \lambda$ is big enough. Then, the operator $\left(1-L_{\lambda} P_{\lambda} \Phi\right)$ is invertible, that is,

$$
1 \in \rho\left(L_{\lambda} P_{\lambda} \Phi\right)
$$

where $L_{\lambda} P_{\lambda} \Phi \in \mathscr{L}(\mathscr{F})$. Moreover, its inverse $\left(1-L_{\lambda} P_{\lambda} \Phi\right)^{-1}$ is positive and it is given by the Neumann series. In fact, since $\left\|L_{\lambda} P_{\lambda} \Phi\right\| \leq 1$ for $\mathfrak{R} \lambda$ big enough (see the next lemma), the spectral radius, $r\left(L_{\lambda} P_{\lambda} \Phi\right)$, of $L_{\lambda} P_{\lambda} \Phi$ is such that $r\left(L_{\lambda} P_{\lambda} \Phi\right) \leq 1$ (see, e.g., [8, Corollary IV.1.4]), and as a consequence $1-L_{\lambda} P_{\lambda} \Phi$ is invertible. Moreover, its inverse is given by the Neumann series. Therefore, since $L_{\lambda} P_{\lambda} \Phi$ is a positive operator (see the next lemma), $\left(1-L_{\lambda} P_{\lambda} \Phi\right)^{-1}$ is positive at least for $\mathfrak{R} \lambda$ big enough.

Moreover, using the compactness of $L_{\lambda} P_{\lambda} \Phi$ and $P_{\lambda} \Phi L_{\lambda}$ (see the next lemma) and the fact that $1 \in \rho\left(L_{\lambda} P_{\lambda} \Phi\right)$, the resolvent of $\mathscr{A}$ in $\lambda$ is

$$
R(\lambda, \mathscr{A})=\left(\begin{array}{cc}
\left(1-L_{\lambda} P_{\lambda} \Phi\right)^{-1} R\left(\lambda, G_{0}\right) & -\left(1-L_{\lambda} P_{\lambda} \Phi\right)^{-1} L_{\lambda} R\left(\lambda, A_{0}\right) \\
-\left(1-L_{\lambda} P_{\lambda} \Phi\right)^{-1} P_{\lambda} \Phi R\left(\lambda, G_{0}\right) & \left(1-L_{\lambda} P_{\lambda} \Phi\right)^{-1} R\left(\lambda, A_{0}\right)
\end{array}\right)
$$

(see [18, Theorem 2.7] and [7, Theorem II.2.8]). Thus the thesis follows immediately.

For the operators $L_{\lambda}$ and $P_{\lambda}$, defined in the previous section, the following lemma holds. 
Lemma 4.2. The operators $L_{\lambda}$ and $P_{\lambda}$ verify the following property:

(1) $P_{\lambda} \Phi$ has one-dimensional range,

(2) $P_{\lambda} \Phi$ is compact,

(3) $P_{\lambda} \Phi L_{\lambda}$ and $L_{\lambda} P_{\lambda} \Phi$ are positive compact operators,

(4) $\lim _{\mathfrak{R} \lambda \rightarrow+\infty}\left\|L_{\lambda} P_{\lambda} \Phi\right\|=0$.

We do not give the proof of the previous lemma since it is immediate: it is just sufficient to observe that $(1) \Rightarrow(2) \Rightarrow(3)$. Moreover, (1) and (4) follow from the definition of the two operators.

As we said before, since $(\mathscr{T}(t))_{t \geq 0}$ is a positive semigroup on the Banach Lattice $\mathscr{E}$ and this is an AL-space, the following result is immediate from classical result.

Corollary 4.3. The growth bound of $(\mathscr{T}(t))_{t \geq 0}, \omega_{0}(\mathscr{T}(\cdot))$, and the spectral bound of $\mathscr{A}$, $s(\mathscr{A})$, are such that

$$
\omega_{0}(\mathscr{T}(\cdot))=s(\mathscr{A})
$$

Moreover, $s(\mathscr{A}) \in \sigma(\mathscr{A})$.

By the previous corollary, it is clear that if we want to find conditions such that the semigroup $(\mathscr{T}(t))_{t \geq 0}$ decays exponentially, it is sufficient to find conditions such that the spectral bound of its generator $\mathscr{A}$ is strictly negative. Information on $s(\mathscr{A})$ can be obtained using the stability results of Engel on two-sided coupled operator (see, e.g., [6]). Important in this sense is the next lemma.

Lemma 4.4 (see, e.g., [14]). Let $\lambda \in \mathbb{C}$ be such that $\mathfrak{R} \lambda>\max \left\{\omega_{0}(\mathcal{U}), \omega_{0}(\mathscr{V})\right\}$. Then, the following statements are true:

(1) $\left(\begin{array}{c}u^{0} \\ f\end{array}\right) \in D(\mathscr{A}) \Leftrightarrow\left(\operatorname{Id}-\mathscr{L}_{\lambda} \Psi\right)\left(\begin{array}{c}u^{0} \\ f\end{array}\right) \in D\left(\mathscr{A}_{0}\right)$,

(2) $(\lambda-\mathscr{A})\left(\begin{array}{c}u^{0} \\ f\end{array}\right)=\left(\lambda-\mathscr{A}_{0}\right)\left(\operatorname{Id}-\mathscr{L}_{\lambda} \Psi\right)\left(\begin{array}{c}u^{0} \\ f\end{array}\right)$, for $\left(\begin{array}{c}u^{0} \\ f\end{array}\right) \in D(\mathscr{A})$.

As an immediate consequence, we obtain the following result.

Theorem 4.5. The operator $A$ can be rewritten in the following way:

$$
\mathscr{A}=\mathscr{A}_{0}\left(\mathrm{Id}-\mathscr{L}_{0} \Psi\right)=\left(\begin{array}{cc}
G_{0} & 0 \\
0 & A_{0}
\end{array}\right)\left(\begin{array}{cc}
\mathrm{Id} & -L_{0} \\
-P_{0} \Phi & \mathrm{Id}
\end{array}\right) .
$$

Moreover,

$$
s(\mathscr{A})<0 \Longleftrightarrow r\left(L_{0} P_{0} \Phi\right)<1 .
$$

Proof. The first part is an easy consequence of the previous lemma (it is sufficient to take $\lambda=0$ in (4.4)), while the second part is a consequence of [7, Theorem VI.3.4] and of the fact that $\omega_{0}\left(G_{0}\right)$ and $\omega_{0}\left(A_{0}\right)$ are strictly negative.

Finally, as a consequence of Corollary 4.3, one can obtain conditions such that the solution of (1.1) decays exponentially. 
3288 Diffusion and delayed birth in an age population equation

Theorem 4.6. If the birth rate $\beta$ and the death rate $\mu$ are such that

$$
\|\beta\|_{\infty} \int_{0}^{\infty} e^{-\int_{0}^{a} \mu(\sigma) d \sigma} d a<\frac{1}{\tau}
$$

then the solution of (1.1) decays exponentially.

Proof. For $F \in \mathscr{F}$ and $\mathfrak{R} \lambda \geq 0$, we have

$$
\begin{aligned}
\left\|L_{\lambda} P_{\lambda} \Phi F\right\|_{L^{1}((-\tau, 0], E)} & =\int_{-\tau}^{0}\left\|L_{\lambda} P_{\lambda} \Phi F(s)\right\|_{E} d s \\
& =\int_{-\tau}^{0} \int_{0}^{\infty}\left|\left(L_{\lambda} P_{\lambda} \Phi F\right)(s, a)\right| d s d a \\
& \leq\|\beta\|_{\infty}\|F\|_{1} \int_{-\tau}^{0} \int_{0}^{\infty}|V(s, 0) U(a, 0)| d a d s \\
& \leq\|\beta\|_{\infty}\|F\|_{1} \int_{-\tau}^{0} \int_{0}^{\infty} e^{-\int_{0}^{-s} \mu(\rho) d \rho} e^{-\int_{0}^{a} \mu(\sigma) d \sigma} d a d s \\
& \leq\|\beta\|_{\infty}\|F\|_{1} \int_{-\tau}^{0} e^{s \mu_{\tau}} d s \int_{0}^{\infty} e^{-\int_{0}^{a} \mu(\sigma) d \sigma} d a \\
& \leq \tau\|\beta\|_{\infty}\|F\|_{1} \int_{0}^{\infty} e^{-\int_{0}^{a} \mu(\sigma) d \sigma} d a .
\end{aligned}
$$

Thus

$$
\left\|L_{\lambda} P_{\lambda} \Phi\right\| \leq \tau\|\beta\|_{\infty} \int_{0}^{\infty} e^{-\int_{0}^{a} \mu(\sigma) d \sigma} d a<1
$$

and $r\left(L_{\lambda} P_{\lambda} \Phi\right)<1$. Recall that $L_{\lambda}, P_{\lambda}, \Phi, \mathcal{U}$, and $\mathscr{V}$ are defined, respectively, in (3.31), (3.34), (2.4), (3.8), and (2.5). In particular, for $\lambda=0$, by Theorem 4.5, it follows that $s(\mathscr{A})<0$. Thus, by Corollary 4.3 , one has that the solution of $(1.1)$ decays exponentially.

Observe that (4.6) is the stability condition obtained by Piazzera in [22].

\section{Acknowledgments}

The author is grateful for the financial support of the Italian "Instituto Nazionale di Alta Matematica" (INdAM). She also wants to thank Klaus Engel and Susanna Piazzera for many helpful discussions.

\section{References}

[1] B. Ainseba and M. Iannelli, Exact controllability of a nonlinear population-dynamics problem, Differential Integral Equations 16 (2003), no. 11, 1369-1384.

[2] S. Aniţa, Analysis and Control of Age-Dependent Population Dynamics, Mathematical Modelling: Theory and Applications, vol. 11, Kluwer Academic, Dordrecht, 2000.

[3] W. Arendt and C. J. K. Batty, Absorption semigroups and Dirichlet boundary conditions, Math. Ann. 295 (1993), no. 3, 427-448.

[4] V. Barbu, M. Iannelli, and M. Martcheva, On the controllability of the Lotka-McKendrick model of population dynamics, J. Math. Anal. Appl. 253 (2001), no. 1, 142-165. 
[5] E. B. Davies, Heat Kernels and Spectral Theory, Cambridge Tracts in Mathematics, vol. 92, Cambridge University Press, Cambridge, 1989.

[6] K.-J. Engel, Positivity and stability for one-sided coupled operator matrices, Positivity 1 (1997), no. 2, 103-124.

[7] Operator Matrices and Systems of Evolution Equations, book manuscript, 1996.

[8] K.-J. Engel and R. Nagel, One-Parameter Semigroups for Linear Evolution Equations, Graduate Texts in Mathematics, vol. 194, Springer, New York, 2000.

[9] G. Fragnelli, Classical solutions for PDEs with nonautonomous past in $L^{p}$-spaces, Bull. Belg. Math. Soc. Simon Stevin 11 (2004), no. 1, 133-148.

[10] G. Fragnelli, P. Martinez, and J. Vancostenoble, Qualitative properties of a population dynamics system describing pregnancy, Math. Models Methods Appl. Sci. 15 (2005), no. 4, 507-554.

[11] G. Fragnelli and D. Mugnai, Nonlinear delay equations with nonautonomous past, preprint.

[12] G. Fragnelli and G. Nickel, Partial functional differential equations with nonautonomous past in $L^{p}$-phase spaces, Differential Integral Equations 16 (2003), no. 3, 327-348.

[13] G. Fragnelli and L. Tonetto, A population equation with diffusion, J. Math. Anal. Appl. 289 (2004), no. 1, 90-99.

[14] G. Greiner, A typical Perron-Frobenius theorem with applications to an age-dependent population equation, Infinite-Dimensional Systems (Retzhof, 1983) (F. Kappel and W. Schappacher, eds.), Lecture Notes in Math., vol. 1076, Springer, Berlin, 1984, pp. 86-100.

[15] J. A.J. Metz and O. Diekmann (eds.), The Dynamics of Physiologically Structured Populations, Lecture Notes in Biomathematics, vol. 68, Springer, Berlin, 1986.

[16] N. V. Minh and N. T. Huy, Characterizations of dichotomies of evolution equations on the halfline, J. Math. Anal. Appl. 261 (2001), no. 1, 28-44.

[17] R. Nagel, Towards a "matrix theory" for unbounded operator matrices, Math. Z. 201 (1989), no. $1,57-68$.

[18] The spectrum of unbounded operator matrices with nondiagonal domain, J. Funct. Anal. 89 (1990), no. 2, 291-302.

[19] R. Nagel and N. T. Huy, Linear neutral partial differential equations: a semigroup approach, Int. J. Math. Math. Sci. (2003), no. 23, 1433-1445.

[20] G. Nickel, Evolution semigroups for nonautonomous Cauchy problems, Abstr. Appl. Anal. 2 (1997), no. 1-2, 73-95.

[21] G. Nickel and A. Rhandi, On the essential spectral radius of semigroups generated by perturbations of Hille-Yosida operators, Tübinger Berichte zur Funktionalanalysis 4 (1994/95), 207-220 (German).

[22] S. Piazzera, An age-dependent population equation with delayed birth process, Math. Methods Appl. Sci. 27 (2004), no. 4, 427-439.

[23] F. Räbiger, R. Schnaubelt, A. Rhandi, and J. Voigt, Non-autonomous Miyadera perturbations, Differential Integral Equations 13 (2000), no. 1-3, 341-368.

[24] H. H. Schaefer, Banach Lattices and Positive Operators, Die Grundlehren der mathematischen Wissenschaften, vol. 215, Springer, New York, 1974.

[25] R. Schnaubelt, Sufficient conditions for exponential stability and dichotomy of evolution equations, Forum Math. 11 (1999), no. 5, 543-566.

[26] G. F. Webb, Theory of Nonlinear Age-Dependent Population Dynamics, Monographs and Textbooks in Pure and Applied Mathematics, vol. 89, Marcel Dekker, New York, 1985.

G. Fragnelli: Dipartimento di Matematica, Università di Roma Tor Vergata, via della Ricerca Scientifica 1, 00133 Roma, Italy

E-mail address: fragnell@mat.uniroma2.it 


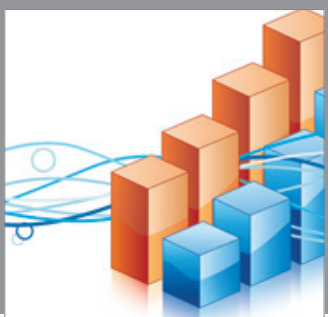

Advances in

Operations Research

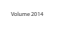

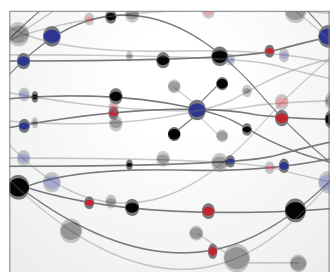

\section{The Scientific} World Journal
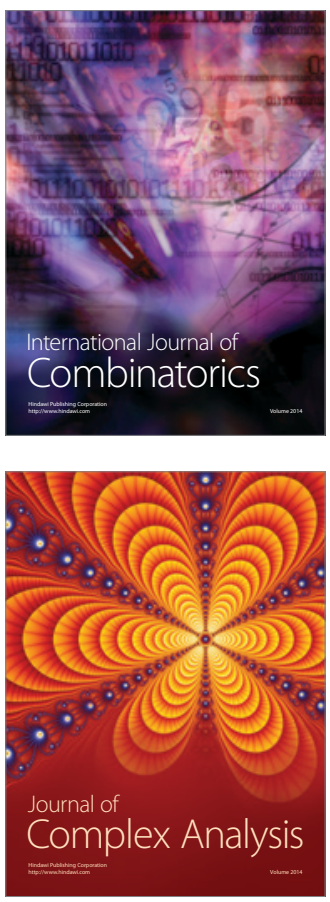

International Journal of

Mathematics and

Mathematical

Sciences
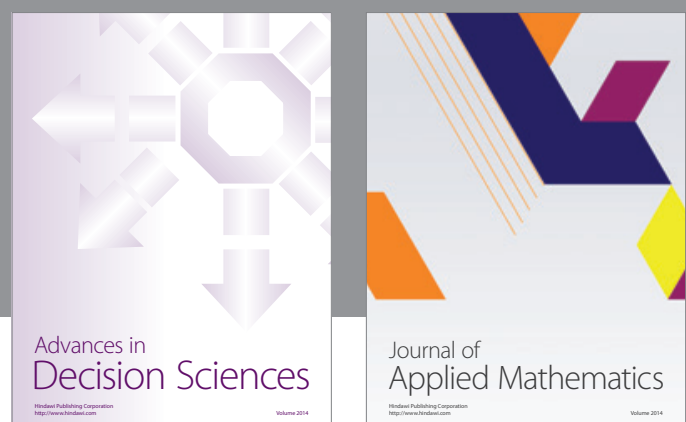

Journal of

Applied Mathematics
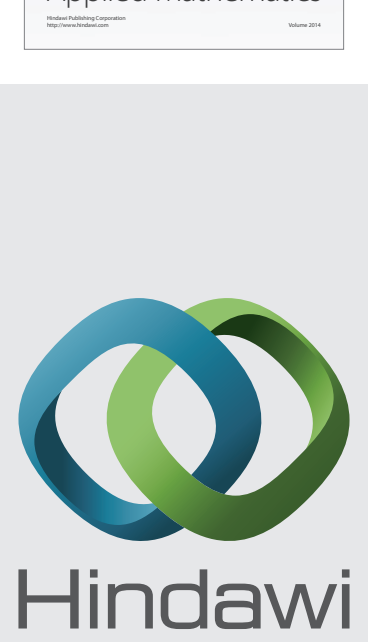

Submit your manuscripts at http://www.hindawi.com
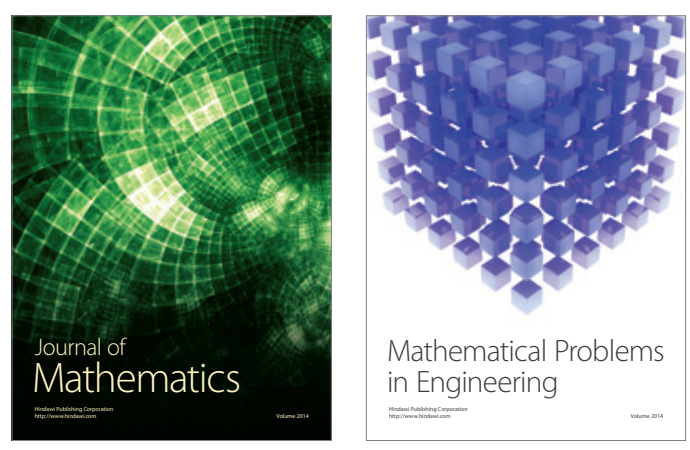

Mathematical Problems in Engineering
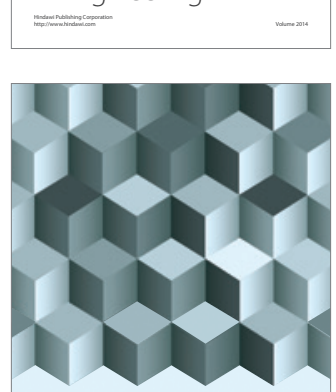

Journal of

Function Spaces
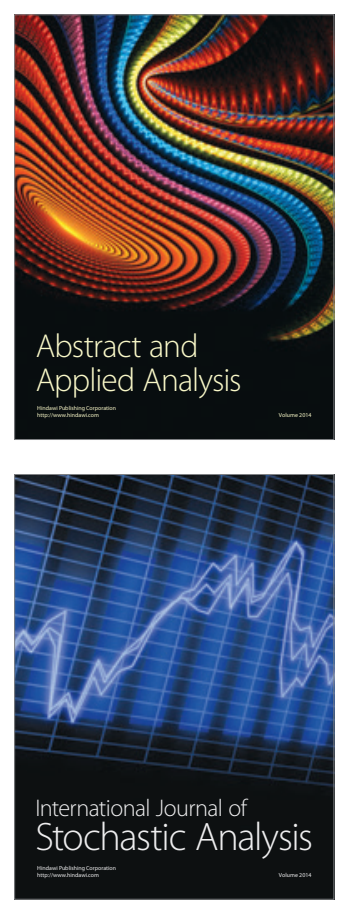

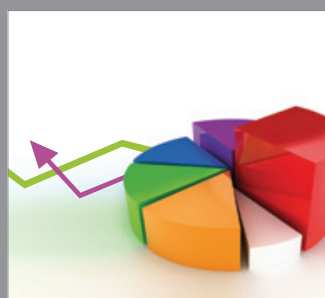

ournal of

Probability and Statistics

Promensencen
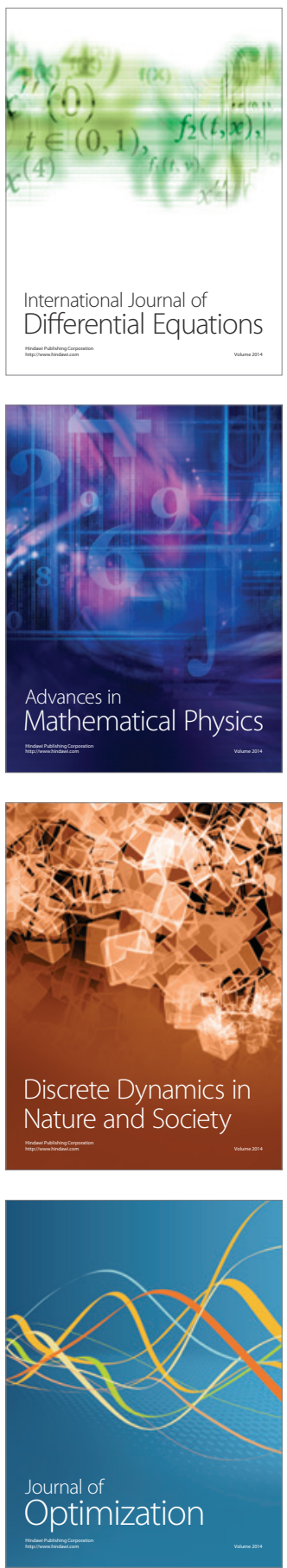